\title{
Recycling of Importin $\alpha$ from the Nucleus Is Suppressed by Loss of RCC1 Function in Living Mammalian Cells
}

\author{
Taro Tachibana ${ }^{1, * *}$, Miki Hieda ${ }^{1}$, Yoichi Miyamoto ${ }^{1}$, Shingo Kose ${ }^{1}$, Naoko Imamoto ${ }^{1}$, and Yoshihiro \\ Yoneda ${ }^{1,2, *}$ \\ ${ }^{1}$ Department of Cell Biology and Neuroscience, Graduate School of Medicine, Osaka University, 2-2 \\ Yamada-oka, Suita, Osaka 565-0871, Japan and ${ }^{2}$ Institute for Molecular and Cellular Biology, Osaka \\ University, 3-1, Yamada-oka, Suita, Osaka 565-0871, Japan
}

\begin{abstract}
We previously reported that the nuclear import of substrates containing SV40 $\mathbf{T}$ antigen nuclear localization signal (NLS) was suppressed in a temperature-sensitive RCC1 mutant cell line, tsBN2, at nonpermissive temperature. Moreover, it was shown that import into wild type BHK21 cell-derived nuclei gradually decreased in heterokaryons between the tsBN2 and BHK21 cells, although the BHK21 nuclei retained wild type RCC1 and should contain RanGTP (Tachibana et al., 1994). In this study, it was found that in the heterokaryons cultured at non-permissive temperature, endogenous importin $\alpha$ was not detected immunocytochemically in the cytoplasm or BHK21 nuclei but only in the tsBN2 nuclei, suggesting that importin $\alpha$ cannot be exported from the RCC1-depleted nuclei. In fact, importin $\alpha$ microinjected into the nucleus of tsBN2 cells at non-permissive temperature remained in the nucleus. These results strongly support the hypothesis that the recycling of importin $\alpha$ from the nucleus requires nuclear RanGTP. Moreover, it was found that cytoplasmic injection of importin $\alpha$ restored the import of SV40 T-NLS substrates in the BHK21 nuclei but not the tsBN2 nuclei in the heterokaryons. This indicates that the decrease of importin $\alpha$ from the cytoplasm in the heterokaryons leads to a suppression of the efficiency of nuclear import of the T-NLS substrate and provides support for the view that nuclear RanGTP is essential for the nuclear entry of the substrates.
\end{abstract}

Key words: nuclear protein transport/importin $\alpha / \operatorname{Ran} / \mathrm{RCC} 1$

The nucleus, in which the genomic DNA is sequestered and its replication and transcription to RNA occur, contains a double membrane, the nuclear envelope. A variety of molecules including proteins and RNAs, are transported through the nuclear pore complexes (NPCs) of the nuclear envelope in both directions in order for the cytoplasm and the nucleus to communicate. The nuclear pore contains an aqueous diffusion channel which allows non-selective passive diffusion of small molecules (<20 40kDa). However, macromolecules larger than $40 \sim 60 \mathrm{kDa}$ can be transported in a signal-

\footnotetext{
* To whom correspondence should be addressed: Department of Cell Biology and Neuroscience, Graduate School of Medicine, Osaka University, 2-2 Yamada-oka, Suita, Osaka 565-0871, Japan.

Tel: +81-6-6879-3210, Fax: +81-6-6879-3219

E-mail: yyoneda@anat3.med.osaka-u.ac.jp

** Present address: Division of Neurochemistry and Neuropharmacology, Department of Neuroscience, Biomedical Research Center, Graduate School of Medicine, Osaka University, 2-2 Yamada-oka, Suita, Osaka 5650871, Japan.

Abbreviations: NLS; nuclear localization signal, NES; nuclear export signal, NPC; nuclear pore complex.
}

and temperature-dependent manner. It has been shown that active nuclear protein import is mediated by nuclear localization signal (NLS), while nuclear protein export is mediated by a nuclear export signal (NES) (for reviews see Mattaj and Englmeier, 1998; Yoneda, 1997).

Conventional NLSs, the first example of which is the simian virus 40 (SV40) large T-antigen, are rich in basic amino acids, and can be classified into two major groups: the single type (for example, the SV40 large T-antigen) and the bipartite type(such as nucleoplasmin and CBP80 (cap binding protein 80) ). The bipartite type contains two clusters of basic amino acids, each of which is separated by approximately 10 amino acids. The basic type NLS-containing karyophiles form a stable complex, termed the nuclear pore-targeting complex via interaction with importin $\alpha / \beta$ to target nuclear pores (Imamoto et al., 1995b). Importin $\alpha$ functions as an NLS-receptor, whereas importin $\beta$ binds to importin $\alpha$, when it is also bound to the NLS substrate and targets the complex to the nuclear pore by interacting with 
components of the NPC (Adam and Adam., 1994; Enenkel et al., 1995; Görlich et al, 1995; Imamoto et al., 1995a; Moroianu et al., 1995). After the targeting of the heterotrimer to the NPC, the complex translocates through the NPC, with the aid of additional soluble factors, a small GTPase Ran and its interacting protein, p10/NTF2 (Moore and Blobel 1994; Paschal and Gerace, 1995). The direct binding of the GTP-bound form of Ran (RanGTP) to importin $\beta$ after translocation of the trimeric complex triggers the dissociation of the complex, thus releasing the NLS-substrate inside the nucleus (Görlich et al., 1996; Moroianu et al., 1996; Rexach et al., 1995). In addition to the basic type NLS-mediated nuclear import pathway, it is known that the import of heterogeneous nuclear RNA-binding protein A1 (hnRNP A1) is mediated by a distinct NLS, referred to as M9, which is rich in glycine and aromatic residues. M9containing substrates are transported into the nucleus by transportin, an importin $\beta$-related transport factor, through direct binding without a requirement for adaptor molecules such as importin $\alpha$. This pathway also requires Ran (Nakielny et al., 1996; Pollard et al., 1996).

Although the yeast, Saccharomyces cerevisiae, is known to produce one importin $\alpha$ homologue, Srp1p, which is an essential gene product (Yano et al., 1992), the family molecules of importin $\alpha$ have been identified in higher eukaryotes. In human, the importin $\alpha$ family can be divided into three distinct subgroups, so-called Rch1 (hSRP1 $\alpha$ ), NPI-1 (hSRP1) and Qip1, which have approximately 50\% amino acid-identity with one another (Köhler et al., 1997; Miyamoto et al., 1997; Tsuji et al., 1997). It has been reported that the SV40 T-NLS substrates can be transported nearly equally efficiently to the nucleus by Rch1 and NPI-1 in a permeabilized cell-free transport assay (Miyamoto et al., 1997). In contrast, it has been shown that the interferon- $\gamma$ dependent nuclear import of STAT1 (signal transducer and activator of transcription 1) is mediated via complex formation with NPI-1, but not Rch1, and importin $\beta$ (Sekimoto et al., 1997). Moreover, it has been demonstrated that Qip1 transports DNA helicase Q1-NLS substrates most efficiently into the nucleus (Miyamoto et al., 1997). It has recently been reported that each subfamily of importin $\alpha$ shows a unique expression pattern in various adult mouse tissues. These findings suggest that a complex divergence exists in the function of importin $\alpha$ and that nuclear protein import might be controlled in a tissue-specific manner by importin $\alpha$ family molecules (Kamei et al., 1999).

After the translocation of karyophilic proteins into the nucleus, the transport factors must be recycled back to the cytoplasm in order to transport the next karyophile into the nucleus. It has been demonstrated that importin $\alpha$ is exported from the nucleus by the cellular apoptosis susceptibility gene product (CAS) (Kutay et al., 1997). CAS is also related to importin $\beta$ and forms a heterotrimeric complex with importin $\alpha$ and RanGTP in the nucleus. It is speculated that the trimer moves from the nucleus to the cytoplasm without
GTP hydrolysis of Ran and that the GTP hydrolysis in the cytoplasm triggers the release of importin $\alpha$ from CAS.

A small GTPase Ran is a key molecule in the nucleocytoplasmic transport of proteins. Ran is located predominantly in the nucleus, but small amounts also exist in the cytoplasm (Bischoff and Ponstingl, 1991b). Ran functions through cycling between GDP- and GTP-bound form and shuttling between the cytoplasm and the nucleus (Hieda et al., 1999). It has been shown that $\mathrm{p} 10 / \mathrm{NTF} 2$ is involved in the recycling of RanGDP from the cytoplasm to the nucleus (Ribbeck et al., 1998; Smith et al., 1998), and acts as a GDP-dissociation inhibitor of RanGDP (RanGDI) (Yamada et al., 1998). It is known that RCC1, which is the sole GDP-GTP exchange factor (GEF) of Ran (Bischoff and Ponstingl, 1991a), is located on chromatin, whereas RanGAP1, which is the sole Ran GTPase activating protein (Bischoff et al., 1994), is located in the cytoplasm and its SUMO (small ubiquitin-like modifier)-1-modified form is located on the cytoplasmic fibers which extend from the NPC (Mahajan et al., 1998; Matunis et al., 1998). The asymmetric distribution of these two factors across the nuclear envelope suggests that nuclear Ran is predominantly the GTP-bound form and cytoplasmic Ran the GDP-bound form, which could assure the directional movement of proteins through the NPC (Görlich et al., 1996; Izaurralde et al., 1997). In addition to RCC1 and RanGAP1, Ran is known to interact with many other molecules, such as RanBP1 and RanBP2. It was recently demonstrated that the binding of RanBP1 to the Ran/importin $\beta$ complex leads to the dissociation of the complex in the cytoplasm, in order for Ran to be recycled to the nucleus, which is an essential feature of nuclear protein import (Bischoff and Görlich, 1997; Hieda et al., 1999).

In our previous study, using a temperature-sensitive RCC1 mutant cell line, tsBN2, we found that the loss of RCC1 function results in the suppression of nuclear protein import efficiency in living cells (Tachibana et al., 1994). In order to examine whether the supply of wild type RCC1 leads to recovery of nuclear import efficiency, we next constructed heterokaryons between tsBN2 cells and wild type BHK21 cells and then injected SV40 T-NLS substrates into the cytoplasm of the heterokaryons. Unexpectedly, it was found that nuclear import into BHK21-derived nuclei as well as tsBN2-derived nuclei gradually decreased after fusion at non-permissive temperature, although the BHK21 nuclei still contained RCC1 protein. In the same study, we postulated that, in the case of tsBN2 cells cultured at nonpermissive temperature, an inhibitory factor could accumulate in the cytoplasm. However, this factor was not identified and the inhibitory mechanism was not clearly elucidated.

In this study, in order to elucidate the molecular basis for the above phenomena observed in heterokaryons, we attempted to examine the intracellular distribution of transport factors in the heterokaryons. As a result, our findings showed that endogenous importin $\alpha$, but not importin $\beta$, 
strongly accumulates only in tsBN2 nuclei, which results in the decrease of cytoplasmic importin $\alpha$. The nuclear export of importin $\alpha$ injected into the tsBN2 nuclei was inhibited, which is consistent with the hypothesis that importin $\alpha$ is recycled back to the cytoplasm by means of complex formation with CAS and RanGTP in the nucleus. This provides the in vivo confirmation for the requirement of nuclear RanGTP in the nuclear export of importin $\alpha$, and the export of import $\alpha$ is essential for the nuclear import of conventional NLS-containing karyophiles to occur normally.

\section{Materials and Methods}

\section{Cell culture and cell fusion}

The tsBN2 cell line is a temperature-sensitive mutant derived from the BHK21 cell line that has a point mutation in RCC1 gene (Uchida et al., 1990). TsBN2 and BHK21 cells were grown in Dulbecco's modified Eagle's minimum essential medium (DMEM; Gibco BRL, MD, USA) supplemented with $5 \%$ fetal bovine serum at $37^{\circ} \mathrm{C}$ (BHK21) or $33.5^{\circ} \mathrm{C}$ (tsBN2). For experiments using heterokaryons (Figs. 1, 2, and 6), tsBN2 cells were co-cultured with BHK21 cells at $33.5^{\circ} \mathrm{C}$ for 48 hours in isoleucine-free minimum essential medium supplemented with $5 \%$ dialyzed serum, transferred to $40^{\circ} \mathrm{C}$, and incubated for 6 hours. They were then fused by the Hemagglutinating Virus of Japan (HVJ, Sendai Virus) as described previously (Tachibana et al., 1994). The tsBN2-BHK21 heterokaryons were further incubated for 6 hours at $40^{\circ} \mathrm{C}$ prior to microinjection.

\section{Microinjection}

Microinjection experiments were performed essentially as described previously (Yoneda et al., 1987). After microinjection and incubation, the cells were fixed with $3.7 \%$ paraformaldehyde in PBS (137 mM NaCl, $2.7 \mathrm{mM} \mathrm{KCl}, 8.1 \mathrm{mM} \mathrm{Na} 2 \mathrm{HPO}_{4}, 1 \mathrm{mM}$ $\mathrm{KH}_{2} \mathrm{PO}_{4}, \mathrm{pH}$ 7.2) for 15 minutes at room temperature. The injected fluorescent labeled proteins were detected by Axiophoto microscopy (Carl Zeiss, Inc. NJ, USA).

\section{Antibodies}

Rabbit anti-mouse importin $\alpha$ (PTAC58/Rch1) antibodies were prepared as described previously (Imamoto et al., 1995a). Fulllength recombinant human RCC1 protein was used to immunize rabbits. Anti-RCC1 antibodies were purified from antiserum on recombinant RCC1-conjugated Sepharose.

\section{Preparation of transport substrates}

To prepare fluorescein isothiocyanate (FITC)-labeled SV40 T-antigen NLS conjugate (FITC-T-BSA), the synthetic peptides (SV40 T; CYGGPKKKRKVEDP) were chemically conjugated with FITC-labeled BSA as described previously (Tachibana et al., 1994).

\section{Expression and purification of recombinant nuclear transport factors}

A full length cDNA of mouse Rch1/PTAC58/importin $\alpha 2 / \mathrm{SRP} 1 \alpha /$ $\alpha-P 1$, mouse NPI-2/importin- $\alpha 7 / \alpha-S 2$ or mouse Qip1/importin-

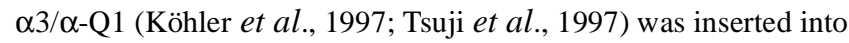
BamHI and EcoRI sites of pGEX-2T (Rch1) or pGEX-6P-1 (NPI2, Qip1). Recombinant mouse Rch1, NPI-2 and Qip1 proteins were prepared from $E$. coli in the same manner as described previously (Imamoto et al., 1995a).

A full length cDNA of mouse Rch1/PTAC58/importin $\alpha 2 /$ SRP1 $\alpha / \alpha-\mathrm{P} 1$ was inserted into BamHI and EcoRI sites of pGEX6P-2-hGFP. Recombinant GST-GFP-Rch1 fusion protein was expressed and purified in the same manner as GST-RanGAP1 (see below). For preparation of GFP-Rch1 fusion protein, GST-GFPRch1 was cleaved by PreScission Protease and purified by chromatography on MonoQ (Amersham Pharmacia, NJ, USA).

CAS was prepared as described previously (Hieda et al., 1999).

Mutation (Q69L) within Ran was created using QuikChange site-directed mutagenesis kit (STRATAGENE). RanQ69L fragment was inserted into the BamHI and EcoRI sites of pGEX-6P-2. Recombinant GST-RanQ69L fusion protein was expressed in $E$. coli as GST-RanGAP1 (see below). An affinity column for the binding assay (described below) was prepared by saturating glutathione-Sepharose with GST-fused protein from the E. coli lysate.

Human RanGAP1 gene (Bischoff et al., 1995) was amplified from a HeLa cell cDNA library by PCR using the synthetic oligonucleotide primers, 5'-GTTCCGGGATCCATGGCCTCGGAAGACATTGCCAAG-3' and 5'-GTTGCAGAATTCCTAGACCTTGTACAGCGTCTGCAG-3'. The PCR product was inserted into the BamHI and EcoRI sites of pGEX-2T vector (Amersham Pharmacia, NJ, USA). Recombinant GST-RanGAP1 protein was expressed by $1 \mathrm{mM}$ IPTG for 12 hours at $20^{\circ} \mathrm{C}$ in E. coli strain BL21(DE3). E. coli cells were harvested by centrifugation and resuspended in $25 \mathrm{ml}$ of TE buffer (50 mM Tris- $\mathrm{HCl}, \mathrm{pH} 8.3,1 \mathrm{mM}$ EDTA, $2 \mathrm{mM}$ DTT) containing $500 \mathrm{mM} \mathrm{NaCl}$ and $1 \mathrm{mM}$ PMSF. Cells were disrupted by means of a french press. The extract was clarified by centrifugation ( 30 minutes at $15,000 \mathrm{~g}$ ) and incubated with $1.5 \mathrm{ml}$ of glutathione-Sepharose. The recombinant protein trapped by the glutathione-Sepharose was eluted with TE buffer containing $100 \mathrm{mM} \mathrm{NaCl}$ and $10 \mathrm{mM}$ glutathione. The GST portion of the GST-RanGAP1 fusion protein was cleaved by a 2 hour incubation at room temperature with $1 \mathrm{NIH}$ unit of thrombin per $100 \mathrm{mg}$ of protein. GST and thrombin were separated from the recombinant protein on a MonoQ column at flow rate of $0.5 \mathrm{ml} / \mathrm{min}$ with a linear gradient from 0.05 to $1.0 \mathrm{M} \mathrm{NaCl}$ in $20 \mathrm{mM}$ Hepes (pH 7.3) and 2 mM DTT. Peak fractions containing RanGAP1 were pooled and dialyzed against $20 \mathrm{mM}$ Hepes (pH 7.3), $110 \mathrm{mM}$ potassium acetate and $2 \mathrm{mM}$ DTT.

\section{Solution binding assay}

Ten $\mu 1$ of immobilized RanQ69L-GTP (see above) was mixed with recombinant GFP-CAS (300 pmol) in addition to recombinant Rch1/PTAC58, NPI-2 or Qip1 (300 pmol), respectively, and the to- 
tal reaction volume was adjusted to $500 \mu \mathrm{l}$ with transport buffer (TB; $20 \mathrm{mM}$ HEPES, pH7.3, $110 \mathrm{mM}$ potassium acetate, $2 \mathrm{mM}$ magnesium acetate, $5 \mathrm{mM}$ sodium acetate, $0.5 \mathrm{mM}$ EGTA, $2 \mathrm{mM}$ DTT). After incubation for $15 \mathrm{~min}$ at room temperature, materials which were bound to the immobilized protein were washed with TB and eluted with TB (pH8.3) containing $10 \mathrm{mM}$ glutathione. Eluted proteins were analyzed by SDS-PAGE followed by Coomassie staining.

\section{Indirect Immunofluorescence}

Cells were washed twice in PBS and fixed with $3.7 \%$ formaldehyde in PBS for 15 minutes at room temperature. After permeabilization with $0.5 \%$ Triton X-100 in PBS for 5 minutes at room temperature, cells were incubated with $10 \mu \mathrm{g} / \mathrm{ml}$ of affinity-purified antibodies for 1.5 hour at room temperature. Rabbit antibodies were detected with FITC- or Cy3-conjugated goat antibodies to rabbit IgG. The samples were examined using an Axiophoto microscope.

\section{Results}

\section{Endogenous importin $\alpha$ accumulates in tsBN2 nuclei of the tsBN2-BHK21 heterokaryon, cultured at non- permissive temperature}

In a previous report, we showed that SV40 T-NLS substrates containing a small number (average 3-5) of peptides per BSA (FITCT-BSA) did not efficiently accumulate in the nuclei of tsBN2 cells when cultured at non-permissive temperature and in which the RCC1 function was lost. Moreover, it was found that in heterokaryon between tsBN2 and wild type BHK21 cells cultured at non-permissive temperature, the import of the FITC-T-BSA into the BHK21-derived nuclei gradually decreased after fusion as well (Tachibana et al., 1994) and Figure 1. Since it is generally thought

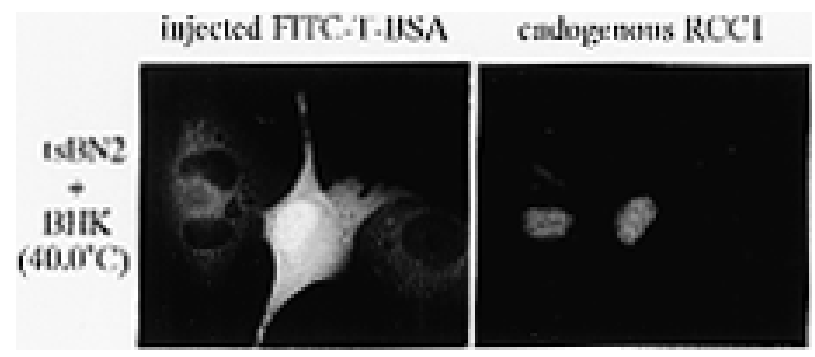

Fig. 1. Nuclear import of SV40 T-antigen NLS containing substrate (FITC-T-BSA) microinjected into tsBN2-BHK21 heterokaryon cultured at non-permissive temperature $\left(40^{\circ} \mathrm{C}\right)$. After complete disappearance of RCC1 from the tsBN2-derived nuclei by incubation for 6 hours at $40^{\circ} \mathrm{C}$, the tsBN2 cells were fused with BHK21 cells as described in the Materials and Methods. After further incubation for 6 hours at $40^{\circ} \mathrm{C}$, FITC-T-BSA was microinjected into the cytoplasm of the tsBN2-BHK21 heterokaryon (left), non-fused BHK21 (middle) and tsBN2 (right). Microinjected cells were then incubated at $40^{\circ} \mathrm{C}$ for $20 \mathrm{~min}$ and fixed. The subcellular localization of endogenous RCC1 was determined by indirect immunofluorescence. that RanGDP can be converted to the GTP-bound form in the BHK21 nuclei, which contain intact RCC1, the results of the fusion experiment cannot be explained by the loss of nuclear RanGTP. Although it has been speculated that a factor that suppresses nuclear import might accumulate in the cytoplasm of heterokaryons after fusion, we were unable to detect inhibitory activity in the cytoplasmic extracts prepared from tsBN2 cells cultured at non-permissive temperatures (data not shown).

Therefore, in order to better understand the nature of the suppression of the import of FITC-T-BSA into BHK21 nuclei in the heterokaryon, the distribution of nuclear import factors in the heterokaryon was examined by using indirect immunofluorescence in conjunction with specific antibodies. As shown in Figure 2, importin $\alpha$ is distributed throughout the cytoplasm and the nucleus at permissive temperature, which is consistent with a previous report (Imamoto et al., 1995a). In heterokaryons cultured at non-permissive temperature, however, endogenous importin $\alpha$ substantially accumulated only in tsBN2 nuclei but was present at negligible or undetectable levels immunocytochemically in the cytoplasm and BHK21 nuclei.

As a result, we then examined the localization of importin $\alpha$ in non-fused tsBN2 cells. As shown in Figure 3, endogenous importin $\alpha$ was detected throughout the cytoplasm and the nucleus at permissive temperature, but only in the nucleus at non-permissive temperature. In contrast, significant accumulation of importin $\beta$ in the nuclei of tsBN2 cells was not observed under the same conditions (data not shown), which is consistent with a previous report (Kose et al., 1999) (see Discussion).

\section{Microinjected importin $\alpha$ is not exported from the nucleus to the cytoplasm in cells in which nuclear RanGTP is depleted}

In the case of tsBN2 cells cultured at non-permissive temperature, inactivation of endogenous mutated RCC1 appears to cause a decline in nuclear RanGTP. It has been shown, using a permeabilized cell-free system, that the nuclear export of importin $\alpha$ is mediated by one of the importin $\beta$ family molecules, CAS, in a RanGTP-dependent manner. Therefore, it is reasonable to assume that the depletion of nuclear RanGTP of tsBN2 cells via the loss of RCC1 function leads to the inhibition of the export of importin $\alpha$ from the nuclei. To test this hypothesis, we analyzed the nuclear export of importin $\alpha$ using a microinjection method in conjunction with live mammalian cells. Recombinant green fluorescent protein (GFP)fused importin $\alpha$ (mouse PTAC58/Rch1) was expressed and purified from $E$. coli. This fusion protein supports nuclear protein import of the NLS-containing substrate in the in vitro import assay (data not shown). After the microinjection of GFP-importin $\alpha$ into a nucleus of tsBN2 cells, the intracellular localization of injected GFP-importin $\alpha$ was examined. As shown in Figure 4, when injected into the nucleus of tsBN2 cells cultured at permissive temperature, GFP-importin $\alpha$ was distributed throughout both the cytoplasm and the nucleus within $30 \mathrm{~min}$. This pattern is similar to that of endogenous importin $\alpha$ (see Fig. 3). By contrast, in the case of tsBN2 cells cultured at the non-permissive temperature, nuclear 


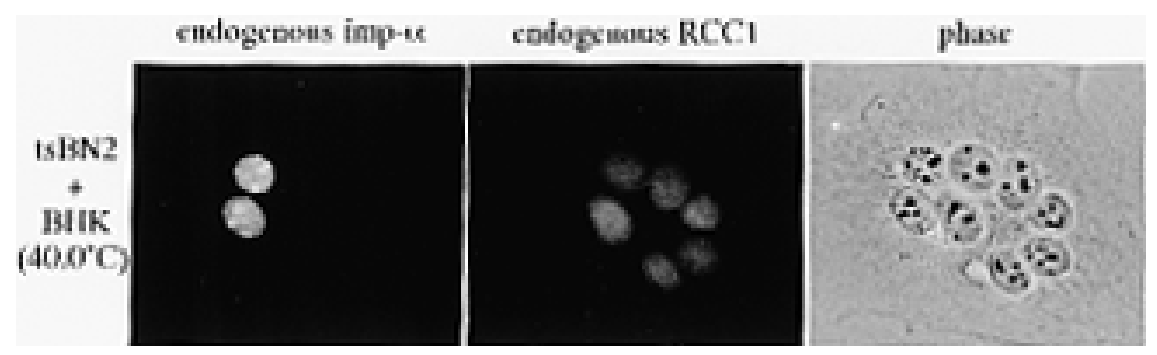

Fig. 2. Subcellular localization of importin $\alpha$ in the tsBN2-BHK21 heterokaryon cultured at non-permissive temperature $\left(40^{\circ} \mathrm{C}\right)$. Under the same condition as in Figure 1, the tsBN2-BHK21 heterokaryons were processed for immunofluorescence staining with affinity-purified anti-mouse importin $\alpha$ (Rch1/ PTAC58) and anti-human RCC1 antibodies as described in Materials and Methods. The tsBN2-derived nuclei show the absence of staining for RCC1.

injected GFP-importin $\alpha$ was not exported from the nucleus. Moreover, when RanGAP1 was co-injected with GFP-importin $\alpha$ into the nucleus of tsBN2 cells cultured at permissive temperature, the injected GFP-importin $\alpha$ could not be detected in the cytoplasm at all, which is consistent with a previous report using Xenopus oocyte (Izaurralde et al., 1997). These results indicate that the decrease in the level of RanGTP in the nuclei of tsBN2 cells inhibits the recycling of importin $\alpha$, thus causing its nuclear accumulation .

\section{All of the three distinct classes of importin $\alpha$ family form a ternary complex with CAS and RanGTP}

It has been demonstrated that CAS binds to Rch1, one of the importin $\alpha$ family of molecules, only in the presence of RanGTP and mediates the nuclear export of Rch1 (Kutay et al., 1997). Howev$\mathrm{er}$, the issue of whether the nuclear export of other importin $\alpha$ family molecules is also mediated by CAS remains unknown. To address this, we examined the binding of CAS to other classes of the

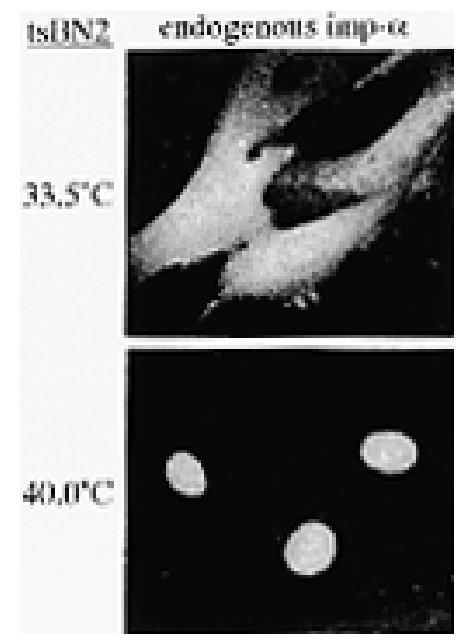

Fig. 3. Subcellular localization of importin $\alpha$ in tsBN2 cells cultured at permissive temperature $\left(33.5^{\circ} \mathrm{C}\right)$ or non-permissive temperature $\left(40^{\circ} \mathrm{C}\right)$. After incubation for 12 hours at $33.5^{\circ} \mathrm{C}$ or at $40^{\circ} \mathrm{C}$, tsBN2 cells were processed for immunofluorescence staining with affinity-purified anti-mouse importin $\alpha$ (Rch1/PTAC58) as described in Materials and Methods. importin $\alpha$ family in the presence of RanGTP. To our knowledge, the mammalian importin $\alpha$ family can be classified into three distinct subclasses (Köhler et al., 1997; Miyamoto et al., 1997; Tsuji et al., 1997). Here, we examined the recombinant mouse Rch1/ PTAC58, NPI-2 (having $81 \%$ amino acid identity to NPI-1) and Qip1 for CAS binding activity in a solution binding assay. The recombinant CAS and each importin $\alpha$ family molecule were mixed and incubated with the immobilized GST-fused RanGTP (GTPasedeficient Q69L mutant) and the bound proteins were analyzed by SDS-PAGE. As shown in Figure 5, all of the three importin $\alpha$ mol-

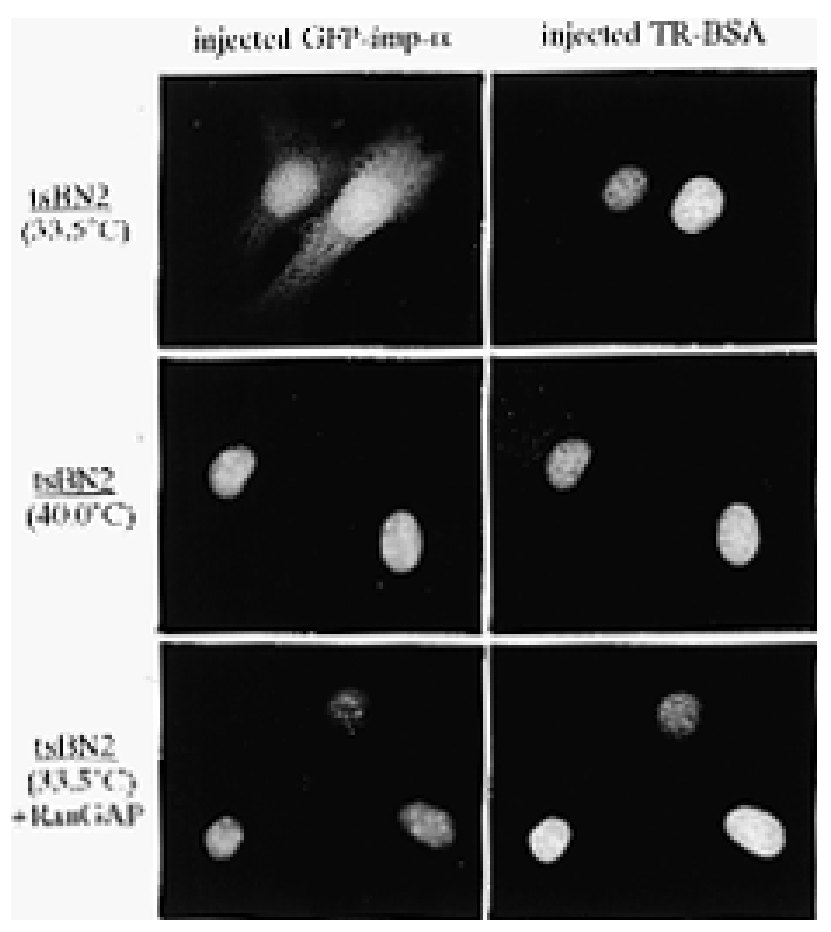

Fig. 4. Analysis of nuclear export of importin $\alpha$ in tsBN2 cells. $0.5 \mathrm{mg} /$ $\mathrm{ml}$ of recombinant GFP-importin $\alpha$ (mouse Rch1/PTAC58) and Texas Redconjugated BSA (TR-BSA) were microinjected with or without $1 \mathrm{mg} / \mathrm{ml}$ RanGAP1 into the nuclei of tsBN2 cells cultured at $33.5^{\circ} \mathrm{C}$ or at $40^{\circ} \mathrm{C}$ for 24 hours. After microinjection, the tsBN2 cells were incubated for $30 \mathrm{~min}$ and fixed. 


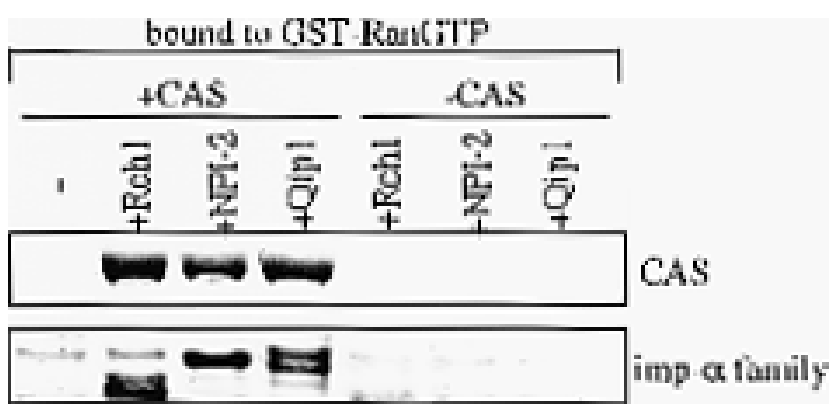

Fig. 5. CAS binds to all three of the distinct subclasses of the importin $\alpha$ family in the presence of RanGTP. Immobilized GTPase-deficient RanQ69L mutant (GTP form) was mixed with recombinant GFP-CAS in the presence of recombinant Rch1/PTAC58, NPI-2 or Qip1, respectively. Bound proteins to immobilized RanQ69L were analyzed by SDS-PAGE followed by Coomassie staining as described in Materials and Methods.

ecules bind to RanGTP only in the presence of CAS. This result indicates that all of the three distinct classes of importin $\alpha$ family form ternary complexes with CAS and RanGTP. Moreover, it was confirmed that the nuclear export of injected GFP-NPI-2 and GFPQip1 was also inhibited in living cells by the loss of nuclear RanGTP using tsBN2 cells (data not shown). These results strongly suggest that not only Rch1 but other importin $\alpha$ family molecules also gradually accumulate in the tsBN2-derived nuclei of the tsBN2-BHK21 heterokaryon cultured at non-permissive temperature, resulting in the decrease in the cytoplasmic importin $\alpha$ family molecules.

\section{Co-injected importin $\alpha$ suppresses the decrease of im- port efficiency of FITC-T-BSA into BHK21 nuclei in tsBN2-BHK21 heterokaryon cultured at non-permis- sive temperature}

We attempted to determine whether the cytoplasmic injection of importin $\alpha$ restores the nuclear import efficiency of the NLS-containing substrate in the heterokaryon. It was found that, in the case of the tsBN2-BHK21 heterokaryon cultured at non-permissive temperature, FITC-T-BSA, when co-injected with importin $\alpha$ into the cytoplasm, migrated only into the BHK21 nuclei and not into the tsBN2 nuclei (Fig. 6). These results strongly suggest that the decline in nuclear import efficiency into the BHK21 nuclei is caused by a decrease in importin $\alpha$ from the cytoplasm in the tsBN2-BHK21 heterokaryon and that the defect in transport into the tsBN2 nuclei is primarily caused by a decline in nuclear RanGTP levels, rather than cytoplasmic depletion of importin $\alpha$. In fact, exogenously injected importin $\alpha$ had no effect on nuclear import in non-fused tsBN2 cells cultured at non-permissive temperature (data not shown). These findings confirm that the nuclear import of SV40 T-NLS containing substrates depends on cytoplasmic importin $\alpha$ in living cells and that nuclear RanGTP is essential for the nuclear import of the SV40 T-NLS substrates.

\section{Discussion}

Previously, we encountered phenomena indicating that, when the SV40 T-NLS substrate containing a small number of NLS peptides was injected into the cytoplasm of heterokaryons between tsBN2 and wild type BHK21 cells cultured at non-permissive temperature, the import efficiency into the BHK21 nuclei also gradually decreased after fusion, even though RCC1 protein was contained in the BHK21 nuclei. These phenomena cannot be explained by the depletion of nuclear RanGTP, since BHK21 nuclei should be able to exchange guanine nucleotides of Ran. In this study, we attempted to better understand this phenomenon in heterokaryons. Through these experiments, we were able to determine the behavior and function of the importin $\alpha$ family of molecules more clearly in living mammalian cells.

\section{Importin $\alpha$ is exported from the nucleus in a nuclear RanGTP-dependent manner}

It has been shown that the nuclear export of importin $\beta$ does not require Ran-dependent GTP hydrolysis. Recently, it was reported that importin $\beta$ can be recycled from the nucleus alone in a Ran-independent manner and its export is mediat-

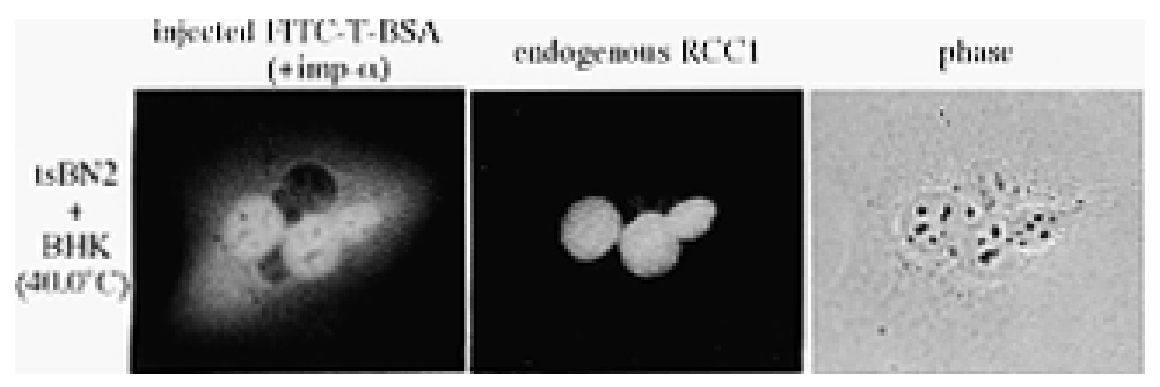

Fig. 6. Effect of co-injected importin $\alpha$ on the nuclear import of FITC-T-BSA in tsBN2-BHK21 heterokaryon cultured at non-permissive temperature $\left(40^{\circ} \mathrm{C}\right)$. Under the same conditions as in Figure 1, FITC-T-BSA was co-injected with $3 \mathrm{mg} / \mathrm{ml}$ of importin $\alpha$ (mouse Rch1/PTAC58) into the cytoplasm of the tsBN2-BHK21 heterokaryons. Microinjected heterokaryons were then incubated at $40^{\circ} \mathrm{C}$ for 20 min and then fixed. The subcellular localization of endogenous RCC1 was determined by indirect immunofluorescence. The tsBN2-derived nuclei showed the absence of staining for RCC1. 
ed by the NPC-binding domain of this molecule (Kose et $a l .$, 1999). This study not only provides support for the view that Ran-dependent GTP hydrolysis is not required for the nuclear export of importin $\beta$ but also indicates that nuclear RanGTP is not essential for its export. On the other hand, more recently it was demonstrated via use of a monoclonal antibody against the C-terminal portion of Ran that Ran/importin $\beta$-related protein complex is exported as a complex from the nucleus in living cells (Hieda et al., 1999). Based on these and other results, we propose that importin $\beta$ is recycled from the nucleus in two distinct ways, i.e., in Randependent and -independent manners.

Meanwhile, with respect to the recycling of importin $\alpha$, it was demonstrated, by use of the in vitro system, that the nuclear export of importin $\alpha$ occurs in the presence of RanGTP, forming an importin $\alpha / \mathrm{CAS} / \mathrm{RanGTP}$ complex. It was found that importin $\alpha$ was mislocalized to the nucleus in the RCC1 homologue prp20 mutant of yeast (Koepp et al., 1996), suggesting that the exchange of GDP for GTP on Ran is necessary for the proper exit of importin $\alpha$ from the nucleus. In addition, in Xenopus laevis oocyte injection experiments, it was shown that the nuclear export of importin $\alpha$ requires the presence of nuclear RanGTP but not the hydrolysis of GTP (Izaurralde et al., 1997).

In support of these findings, the study described here shows that endogenous importin $\alpha$ became localized predominantly in the tsBN2-derived nuclei in heterokaryons between tsBN2 and BHK21 cells cultured at non-permissive temperature, whereas it was distributed throughout the cytoplasm and nucleus at permissive temperature. Moreover, importin $\alpha$, when microinjected into the tsBN2-derived nuclei was found not to be exported in the heterokaryons as well as the non-fused tsBN2 cells at non-permissive temperature. In contrast, it was reported that a significant accumulation of endogenous importin $\beta$ was not observed in tsBN2 cells cultured at non-permissive temperature, and that the nuclear export of importin $\beta$ occurred in tsBN2 cell incubated at non-permissive temperature (Kose et al., 1999), while the export of the leucine-rich NES-containing substrates was inhibited at the identical assay conditions. These results strongly suggest that nuclear RanGTP is essential for the nuclear export of importin $\alpha$, but not importin $\beta$; that is, importin $\alpha$ is recycled from the nucleus only in a Ran-dependent manner.

Furthermore, exogenously introduced importin $\alpha$ restored the import activity of the SV40 T-NLS substrates into the BHK21-derived nuclei in the heterokaryons, providing clear in vivo evidence that cytoplasmic importin $\alpha$ is crucial for the nuclear import of NLS-substrates. On the other hand, the import into the tsBN2-derived nuclei, which do not contain RCC1 protein, in the same heterokaryons was not restored by cytoplasmic injection of importin $\alpha$, indicating that the nuclear entry of the SV40 T-NLS substrates requires nuclear RanGTP.

\section{Complex formation of all the importin $\alpha$ family mole- cules with CAS and RanGTP}

It is known that, in mammalian cells, the importin $\alpha$ family consists of at least three distinct subclasses which have about $50 \%$ amino acid identity with one another. Functional divergence among all these molecules has been revealed. In this study, the issue of whether all the three family molecules form a complex with CAS and RanGTP was addressed. As shown in Figure 5, it was found that they were precipitated by RanGTP only in the presence of CAS in a solution binding assay. These results may not completely exclude the possibility that a CAS-like carrier molecule but not CAS itself binds to and exports importin $\alpha$ family molecules other than Rch1. However, it is certain that all the importin $\alpha$ molecules responsible for the import of SV40TNLS substrates are not recycled back from the RanGTP-depleting nucleus, because the decrease of the import efficiency of the SV40 T-NLS substrates observed in the heterokaryons at non-permissive temperature was suppressed by cytoplasmic injection of importin $\alpha$ molecules.

\section{Importin $\alpha$-dependent nuclear import pathway}

The importin $\alpha / \beta$-dependent pathway was the first pathway identified and has been the most extensively characterized. Subsequently, various import pathways have been identified and characterized. Meanwhile, only a few karyophiles exist for which the import pathway has been identified precisely. Therefore, it is significant to understand the pathway by which individual karyophiles are transported into the nucleus so as to completely understand the entire scheme of intracellular protein traffic between the nucleus and the cytoplasm.

As shown in Figure 1, in the case of heterokaryons between tsBN2 and BHK21 cells cultured at non-permissive temperature, the nuclear import efficiency of SV40 T-NLS substrates containing a small number of NLS peptides was decreased. However, it should be noted that the nuclear import of substrates which contain a large number (average 810) of NLS peptides was only negligibly affected (data not shown), suggesting that importin $\alpha$ family molecules are not completely depleted in the cytoplasm of the heterokaryons. Therefore, although we cannot determine precisely whether the substrates which are transported into the BHK21-derived nuclei of the heterokaryons are mediated by importin $\alpha$ or not, by using this in vivo heterokaryon system we can assume that the import of substrates which are not efficiently imported into the BHK derived nuclei of the heterokaryons are at least in part dependent on importin $\alpha$.

Acknowledgements. We thank Mark Rheaume for his valuable discussion. This work was supported by Grant-in-Aid for Scientific Research on Priority Areas (A) (No. 07282103), Grant-in-Aid for Scientific Research (A) (No. 08558079), Grant-in-Aid for Scientific Research (B) (No. 08458229), Grant-in-Aid for Scientific Research (C) (No. 08680764), 
Grant-in-Aid for COE Research (No. 07CE2006), and Grant-in-Aid for Scientific Research on Priority Areas (B) (No. 11237202) from the Japanese Ministry of Education, Science, Sports and Culture, the Human Frontier Science Program, and the Mitsubishi Foundation.

\section{References}

Adam, E.J. and Adam, S.A. 1994. Identification of cytosolic factors required for nuclear location sequence-mediated binding to the nuclear envelope. J. Cell Biol., 125: 547-555.

Bischoff, F.R. and Görlich, D. 1997. RanBP1 is crucial for the release of RanGTP from importin $\beta$-related nuclear transport factors. FEBS Lett., 419: $249-254$.

Bischoff, F.R., Klebe, C., Kretschmer, J., Wittinghofer, A., and Ponstingl, H. 1994. RanGAP1 induces GTPase activity of nuclear Ras-related Ran. Proc. Natl. Acad. Sci. USA, 91: 2587-2591.

Bischoff, F.R., Krebber, H., Kempf, T., Hermes, I., and Ponstingl, H. 1995. Human RanGTPase-activating protein RanGAP1 is a homologue of yeast Rnalp involved in mRNA processing and transport. Proc. Natl. Acad. Sci.USA, 92: 1749-1753.

Bischoff, F.R. and Ponstingl, H. 1991a. Catalysis of guanine nucleotide exchange on Ran by the mitotic regulator RCC1. Nature, 354: 80-82.

Bischoff, F.R. and Ponstingl, H. 1991b. Mitotic regulator protein RCC1 is complexed with a nuclear ras-related polypeptide. Proc. Natl. Acad. Sci. USA, 88: 10830-10834.

Enenkel, C., Blobel, G., and Rexach, M. 1995. Identification of a yeast karyopherin heterodimer that targets import substrate to mammalian nuclear pore complexes. J. Biol. Chem., 270: 16499-16502.

Görlich, D., Kostka, S., Kraft, R., Dingwall, C., Laskey, R.A., Hartmann, E., and Prehn, S. 1995. Two different subunits of importin cooperate to recognize nuclear localization signals and bind them to the nuclear envelope. Curr. Biol., 5: 383-392.

Görlich, D., Pante, N., Kutay, U., Aebi, U., and Bischoff, F.R. 1996. Identification of different roles for RanGDP and RanGTP in nuclear protein import. EMBO J., 15: 5584-5594.

Hieda, M., Tachibana, T., Yokoya, F., Kose, S., Imamoto, N., and Yoneda, Y. 1999. A monoclonal antibody to the $\mathrm{COOH}$-terminal acidic portion of Ran inhibits both the recycling of Ran and nuclear protein import in living cells. J. Cell Biol., 144: 645-655.

Imamoto, N., Shimamoto, T., Takao, T., Tachibana, T., Kose, S., Matsubae, M., Sekimoto, T., Shimonishi, Y., and Yoneda, Y. 1995a. In vivo evidence for involvement of a $58 \mathrm{kDa}$ component of nuclear pore- targeting complex in nuclear protein import. EMBO J., 14: 3617-3626.

Imamoto, N., Tachibana, T., Matsubae, M., and Yoneda., Y. 1995b. A karyophilic protein forms a stable complex with cytoplasmic components prior to nuclear pore binding. J. Biol. Chem., 270: 8559-8565.

Izaurralde, E., Kutay, U., von Kobbe, C., Mattaj, I.W., and Görlich, D. 1997. The asymmetric distribution of the constituents, of the Ran system is essential for transport into and out of the nucleus. EMBO J., 16: 65356547.

Kamei, Y., Yuba, S., Nakayama, T., and Yoneda, Y. 1999. Three distinct classes of the $\alpha$-subunit of the nuclear pore-targeting complex (importin$\alpha)$ are differentially expressed in adult mouse tissues. J. Histochem. Cytochem., 47: 363-372.

Koepp, D.M., Wong, D.H., Corbett, A.H., and Silver, P.A. 1996. Dynamic localization of the nuclear import receptor and its interactions with transport factors. J. Cell Biol., 133: 1163-1176.

Köhler, M., Ansieau, S., Prehn, S., Leutz, A., Haller, H., and Hartmann, E. 1997. Cloning of two novel human importin- $\alpha$ subunits and analysis of the expression pattern of the importin- $\alpha$ protein family. FEBS Lett., 417: 104-108.

Kose, S., Imamoto, N., Tachibana, T., Yoshida, M., and Yoneda, Y. 1999. $\beta$-Subunit of nuclear pore-targeting complex (importin- $\beta$ ) can be export- ed from the nucleus in a Ran-independent manner. J. Biol. Chem., 274: 3946-3952.

Kutay, U., Bischoff, F.R., Kostka, S., Kraft, R., and Görlich, D. 1997. Export of importin $\alpha$ from the nucleus is mediated by a specific nuclear transport factor. Cell, 90: 1061-1071.

Mahajan, R., Gerace, L., and Melchior, F. 1998. Molecular characterization of the SUMO-1 modification of RanGAP1 and its role in nuclear envelope association. J. Cell Biol., 140: 259-270.

Mattaj, I.W. and Englmeier, L. 1998. Nucleocytoplasmic transport: the soluble phase. Annu. Rev. Biochem., 67: 265-306.

Matunis, M.J., Wu, J., and Blobel, G. 1998. SUMO-1 modification and its role in targeting the Ran GTPase-activating protein, RanGAP1, to the nuclear pore complex. J. Cell Biol., 140: 499-509.

Miyamoto, Y., Imamoto, N., Sekimoto, T., Tachibana, T., Seki, S., Tada, Enomoto, T., and Yoneda, Y. 1997. Differential modes of nuclear localization signal (NLS) recognition by three distinct classes of NLS receptors. J. Biol. Chem., 272: 26375-26381.

Moore, M.S. and Blobel, G. 1994. Purification of a Ran-interacting protein that is required for protein import into the nucleus. Proc. Natl. Acad. Sci. USA, 91: 10212-10216.

Moroianu, J., Blobel, G., and Radu, A. 1995. Previously identified protein of uncertain function is karyopherin $\alpha$ and together with karyopherin $\beta$ docks import substrate at nuclear pore complexes. Proc. Natl. Acad. Sci. USA, 92: 2008-2011.

Moroianu, J., Blobel, G., and Radu, A. 1996. Nuclear protein import: RanGTP dissociates the karyopherin $\alpha / \beta$ heterodimer by displacing $\alpha$ from an overlapping binding site on $\beta$. Proc. Natl. Acad. Sci. USA, 93: 70597062.

Nakielny, S., Siomi, M.C., Siomi, H., Michael, W.M., Pollard, V., and Dreyfuss, G. 1996. Transportin: nuclear transport receptor of a novel nuclear protein import pathway. Exp. Cell Res., 229: 261-266.

Paschal, B.M. and Gerace, L. 1995. Identification of NTF2, a cytosolic factor for nuclear import that interacts with nuclear pore complex protein p62. J. Cell Biol., 129: 925-937.

Pollard, V.W., Michael, W.M., Nakielny, S., Siomi, M.C., Wang, F., and Dreyfuss, G. 1996. A novel receptor-mediated nuclear protein import pathway. Cell, 86: 985-994.

Rexach, M. and Blobel, G. 1995. Protein import into nuclei: association and dissociation reactions involving transport substrate, transport factors, and nucleoporins. Cell, 83: 683-692.

Ribbeck, K., Lipowsky, G., Kent, H.M., Stewart, M., and Görlich, D. 1998. NTF2 mediates nuclear import of Ran. EMBO J., 17: 6587-6598.

Sekimoto, T., Imamoto, N., Nakajima, K., Hirano, T., and Yoneda, Y. 1997. Extracellular signal-dependent nuclear import of Stat1 is mediated by nuclear pore-targeting complex formation with NPI-1, but not Rch1. EMBO J., 16: 7067-7077.

Smith, A., Brownawell, A., and Macara, I.G. 1998. Nuclear import of Ran is mediated by the transport factor NTF2. Curr. Biol., 8: 1403-1406.

Tachibana, T., Imamoto, N., Seino, H., Nishimoto, T., and Yoneda, Y. 1994. Loss of RCC1 leads to suppression of nuclear protein import in living cells. J. Biol. Chem., 269: 24542-24545.

Tsuji, L., Takumi, T., Imamoto, N., and Yoneda, Y. 1997. Identification of novel homologues of mouse importin $\alpha$, the $\alpha$ subunit of the nuclear pore-targeting complex, and their tissue-specific expression. FEBS Lett., 416: $30-34$.

Uchida, S., Sekiguchi, T., Nishitani, H., Miyauchi, K., Ohtsubo, M., and Nishimoto, T. 1990. Premature chromosome condensation is induced by a point mutation in the hamster RCC1 gene. Mol. Cell. Biol., 10: 577584.

Yamada, M., Tachibana, T., Imamoto, N., and Yoneda. Y., 1998. Nuclear transport factor p10/NTF2 functions as a Ran-GDP dissociation inhibitor (Ran-GDI). Curr. Biol., 8: 1339-1342.

Yano, R., Oakes, M., Yamaghishi, M., Dodd, J.A., and Nomura, M., 1992. 
Cloning and characterization of SRP1, a suppressor of temperature-sensitive RNA polymerase I mutations, in Saccharomyces cerevisiae. Mol. Cell. Biol., 12: 5640-5651.

Yoneda, Y. 1997. How proteins are transported from cytoplasm to the nucleus. J. Biochem. (Tokyo)., 121: 811-817.

Yoneda, Y., Arioka, T., Imamoto-Sonobe, N., Sugawa, H., Shimonishi, Y., and Uchida, T. 1987. Synthetic peptides containing a region of SV40 large T-antigen involved in nuclear localization direct the transport of proteins into the nucleus. Exp. Cell Res., 170: 439-452.

(Received for publication, December 28, 1999

and accepted, January 28, 2000) 